\title{
Eingebrannte Bilder
}

\author{
Die Problematik medialer Bildberichterstattung \\ am Beispiel von School Shootings. Von Melanie Verhovnik
}

\begin{abstract}
School Shootings, in den Medien meist als Amokläufe bezeichnet, sind Ereignisse von enormer Tragweite für alle Beteiligten. Die im Artikel präsentierten Ergebnisse einer umfangreichen Studie zur Medienberichterstattung über School Shootings belegen, welchen Umfang Bilder zu School Shootings in Print- und TV-Medien einnehmen, welche Inhalte sie zeigen und wie sie zu beurteilen sind. Parallel dazu durchgeführte Gruppendiskussionen mit Schüler_innen und Studierenden dokumentieren, dass ein zahlenmäßig relativ kleiner Teil der (Bild)Berichterstattung diese dominiert und insbesondere emotionale und emotionalisierende Inhalte von Rezipienten erinnert werden.
\end{abstract}

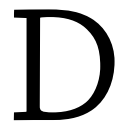
as Jahr 1999 war das zweite Jahr in Folge, in dem in den USA ein School Shooting ${ }^{1}$ „Top Story of the Year“ wurde. Nachdem 1998 das Jonesboro-Shooting von 46 Prozent der Amerikaner „very closely“ verfolgt worden war und das Oregon High School-Shooting auf Platz zwei der Medienereignisse in diesem Jahr landete (46 Prozent „very closely“) (vgl. Pew Research Center for the People and the Press 1999), sprengte das Columbine-Shooting vom 20. April 1999 jegliche Dimensionen bisheriger Berichterstattung über solche Ereignisse.

An diesem Tag starben zwölf Schüler_innen und ein Lehrer der Columbine Highschool durch die Hand ihrer Mitschüler Eric H. (18) und Dylan K. (17). Noch während die Täter in der Schule nach Opfern suchten, waren die Medien vor Ort. Ab 11:30 Uhr zeigten über dem Gelände der Schule kreisende Helikopter

1 School Shootings werden in der medialen Berichterstattung häufig als „Amokläufe“ bezeichnet, obwohl der Bezug zu einem plötzlichen Gewaltausbruch falsch ist. School Shootings sind Ergebnisse längerer, der Tat vorhergehender psychischer, psycho-sozialer und struktureller Prozesse (vgl. Verhovnik 2015, S. 30ff, 95ff, 262ff; im Druck).
Dr. Melanie Verhovnik, Dipl.- Journ., M.A., ist Wissenschaftliche Mitarbeiterin am Studiengang Journalistik der Katholischen Universität EichstättIngolstadt. 
den aktuellen Stand der Dinge, übertrugen erstmalig live, wie Spezialeinsatzgruppen des SWAT-Kommandos versuchten, das Gelände zu sichern, Schülergruppen zu evakuieren und die eintreffenden Notfallambulanzen zu organisieren (vgl. Addington 2009, S. 1427; Larkin 2007, S. 11). Muschert und Larkin (2007, S. 6-7) sprechen von 400 bis 500 Journalisten, die sich noch während bzw. unmittelbar nach der Tat vor Ort einfanden und von dort berichteten. Dazu kamen zwischen 75 und 90 Übertragungswagen und 60 Kameras, die die Bilder von der Tat und den Tätern in alle Welt sendeten.

Das Columbine Shooting wurde von 68 Prozent der Amerikaner "very closely“ verfolgt, von weiteren 24 Prozent "fairly closely" (vgl. Pew Research Center for the People and the Press 1999). Obwohl jüngere Menschen unter 30 Jahren die Nachrichten ansonsten weniger intensiv verfolgen als andere, war das Interesse an der Berichterstattung genau in dieser Gruppe am höchsten. 73 Prozent der unter 30-Jährigen verfolgten die Columbine-Berichterstattung "very closely“, sowie 65 Prozent derjenigen zwischen 30 und 49 Jahren und 69 Prozent derjenigen ab 50 Jahren.

\section{Das Columbine-Shooting als Vorbild für andere Taten}

Wieweit die Nachwirkung dieses „iconic school shootings“ (Newman zitiert nach: Associated Press 2009) gehen würde, konnten die Täter Eric H. und Dylan K. natürlich nicht wissen - was sie nicht davon abhielt, darüber zu spekulieren. Dylan K. sagte in einem von ihm und Eric H. produzierten Video: „Wir werden Nachfolger haben, weil wir so verdammt göttlich sind." Daneben fantasierten die beiden Täter über einen möglichen Spielfilm, den der Regisseur Steven Spielberg über ihre Tat drehen könnte (vgl. Weirauch/Buermann 2010, S. 73).

Und tatsächlich: Die selbstgedrehten Videos, Blogs und Fotos, die von den Columbine-Tätern und Tätern nachfolgender Taten in Umlauf gebracht werden und in einer sehr hohen Anzahl existieren, haben zu einer nicht überschaubaren Fangemeinde, hauptsächlich online, geführt (vgl. u. a. Lindgren 2012; Böckler/ Seeger 2010). Auch aus diesem Grund verfassten einige Täter ihre Videobotschaften oder Tagebucheinträge nicht oder nicht ausschließlich in ihrer Muttersprache, sondern auf Englisch, um ein größeres Publikum zu erreichen. Zeugen oder Aufzeichnungen geben z. B. bei deutschen Taten darüber Auskunft, dass die Täter zum Shooting an der Columbine High School im Internet recherchiert haben. Dies ist für Meißen bekannt (wobei die Aus- 
sagen vage bleiben und schriftliche Beweise nicht existieren), für Erfurt und Winnenden nachgewiesen (Computerrecherchen der Täter) und für Emsdetten durch das Tagebuch des Täters belegt (vgl. Bannenberg 2010, S. 56-58; Polizeidirektion Waiblingen 2009, Szumelda 2007, S. 51, 63). Imitiert wird sowohl die Kleidung (schwarze Handschuhe, umgedrehte Baseball-Kappen, schwarzer Mantel), die eigene Übererhöhung der Täter (Eric H. schrieb in seinen Tagebüchern auf Deutsch "Ich bin Gott") als auch die Schrift (vgl. Bannenberg 2010, S. 57-58). Robert S. (Erfurt 2002) speicherte zwei Dokumente zum Thema Amok und ein Foto vom Columbine-Shooting auf dem Computer seines Vaters, von dem aus er Zugang ins Internet hatte. Das Foto sicherte er unter dem Titel „Durchblick“ (vgl. Robertz 2004, S. 235).

\section{Medien werden instrumentalisiert}

\section{- und lassen dies zu}

Dass School Shootings eine Welle an Veröffentlichungen und öffentlicher Diskussion nach sich ziehen, wird von Tätern inzwischen bei ihren Tatvorbereitungen berücksichtigt (vgl. u. a. Sitzer 2013, S. 298-299; Bondü 2012, S. 67; Twemlow 2002, S. 477). Und Medien kommen dieser Aufforderung nach, steigern sensationalistische Bilder und seitenweise detaillierte Berichterstattung doch die Auflage bzw. die Aufmerksamkeit des Publikums. „In fact, many shooters have been quite media-savy, and have consciously

Die Täter berücksichtigen bei der Vorbereitung ihrer Tat, dass School Shootings eine Welle an Medienberichterstattung nach sich ziehen. used media to convey messages. School shooters are quite certain that they are acting on a public stage, and in fact intend to seize this mediatized stage" (Muschert 2013, S. 168).

Fantasien über das Nachwirken der Tat spielen nicht nur in der Entwicklung von School Shootings eine große Rolle, sondern sind gleichzeitig ein gemeinsames Merkmal der (meisten) Täter (vgl. u. a. Newman 2004, S. 250-251; Moore et al. 2003, S. 118). Cho Seung H., der Täter des School Shootings an der Virginia Tech University, inszenierte sich selbst als Rächer eines Unrechts und Nachfolger der Columbine-Täter. Er schickte ein Paket mit Bildern, Texten und Videos an den Fernsehsender NBC und dieser sendete die Hass-Botschaften in Auszügen (vgl. Meier 2007, S. 2). „Jetzt sind diese Bilder im Cyberspace und können nicht mehr zurückgeholt werden. Cho hat sich als finsterer Held verewigt, nicht im allgemeinen Gedächtnis (in diesem wird Blacksburg nach einigen Monaten unter den Sedimenten der laufend anfallenden News verschwunden sein), aber in den absonderli- 
chen Communities der Randständigen, Verschwörungsgläubigen und Paranoiker. Die Heldenposen des Mörders oder eben ,Märtyrers'von Blacksburg sind, so ist leider anzunehmen, eine neue harte Droge, mit der potentielle Amokläufer und Selbstmordattentäter sich in explosive Gemütszustände versetzen können“ (Meier 2007, S. 2). Ein anderer Täter, Luke W. (Pearl 1997), übergab kurz vor der Tat sein Testament und eine Art Manifest, das seine Tat erklären sollte, einem Freund (vgl. Newman 2004, S. 249). Der Täter des deutschen School Shootings von Emsdetten 2006, Bastian B., schickte alle seine Aufzeichnungen an den Betreiber einer Website, die sich mit School Shootings beschäftigt (vgl. Szumelda 2007, S. 9; Engels 2007, S. 35-56). Auch über andere Wege setzen sich Täter über bildhafte Botschaften für die Medien in Szene: In Deutschland trug der Ansbacher Täter Georg R. (2009) ein T-Shirt mit der Aufschrift "Made in School“, eine klare Verantwortungszuschreibung an die Institution Schule (vgl. Böckler/Seeger/Sitzer 2012, S. 39). Eric Pekka A. (Jokela 2007) trug ein T-Shirt mit der Aufschrift „Humanity is overrated“, ein Beispiel seiner Gedanken- und Gefühlswelt und ein Bezug zum Columbine-Shooting, das er intensiv studiert hatte und dessen Täter Eric H. und Dylan K. sich ebenfalls mit „natürlicher Selektion“ („Natural Selection“ u. a. als T-Shirt bei der Tat getragen) beschäftigten (vgl. Böckler/Seeger/Sitzer 2012, S. 39; Larkin 2007, S. 133).

\section{Die Bildberichterstattung bei School Shootings - Forschungsdesign}

Die folgenden Ergebnisse sind Teil einer Studie, die als Dissertationsprojekt am Lehrstuhl für Journalistik I an der Katholischen Universität Eichstätt-Ingolstadt entstanden ist (Verhovnik 2015, im Druck). Mittels einer Kombination aus qualitativer und quantitativer Inhaltsanalyse wurde einerseits analysiert, wie die deutsche Berichterstattung über School Shootings aussieht, welche Rolle das Columbine-Shooting spielt und welche Deutungsmuster in Hinblick auf Themen, Ursachenzuschreibungen und Lösungsvorschläge über die Medien transportiert werden. Die Stichprobe bestand aus einem Sample deutscher Leitmedien in Print und TV $(\mathrm{N}=2014) .^{2}$ In einem zweiten Schritt wurde eine

2 "Frankfurter Allgemeine Zeitung“ (FAZ), „Süddeutsche Zeitung“ (SZ), "Bild-Zeitung“ (Bild), „Spiegel“, „stern“. Hauptnachrichtensendungen von ARD, ZDF. RTL, Sat.1, Kabel eins und ProSieben. 
sensibilisierte Rezipientengruppe ${ }^{3}$ nach ihren Erinnerungen, Eindrücken, Meinungen und Einstellungen zum Thema School Shootings ${ }^{4}$ befragt. An den zehn Gruppendiskussionen nahmen insgesamt 89 Schüler und Studierende im Alter zwischen 14 und 22 Jahren aus Realschule, Gymnasium und Universität teil.

\section{Ergebnisse der Studie}

Artikel über School Shootings in Zeitungen oder Zeitschriften enthalten überwiegend (63,1 Prozent) kein Foto oder sonstige grafische Elemente. Ein knappes Viertel der Beiträge (22,7 Prozent, $\mathrm{N}=401$ ) besitzt ein beigestelltes grafisches Element, weitere 5,9 Prozent $(\mathrm{N}=105)$ zwei Elemente. Relativ wenige Beiträge sind mit mehr als zwei grafischen Elementen ausgestattet, 2,5 Prozent mit drei Elementen, 2,2 Prozent mit vier Elementen und 3,5 Prozent mit fünf und noch mehr Fotos, Infografiken oder sonstigen Schaubildern. Im Einzelfall handelt es sich hierbei um bis zu 56 (!) einzelne grafische Elemente. Eine Kreuztabelle der grafischen Elemente in Bezug auf das jeweilige Printmedium zeigt, dass eine größere Zahl an Bildern hauptsächlich von der „Bild-Zeitung“ und den Magazinen „Spiegel“ und „Stern“ verwendet wird.

Betrachtet man die Größe der analysierten grafischen Elemente, so war die überwiegende Anzahl (73,8 Prozent, N=896, Mehrfachantworten möglich, da bis zu fünf erhobene Elemente) kleiner als eine viertel Seite. Dies war bei 896 von 1213 analysierten Bildern der Fall. Knapp 15 Prozent $(\mathrm{N}=179)$ waren eine viertel Seite groß, etwas über sechs Prozent zwischen einer viertel und einer halben Seite $(\mathrm{N}=75)$ und knapp zwei Prozent $(\mathrm{N}=24)$ eine halbe Seite groß. Mehr Umfang als eine halbe Zei-

3 Als „sensibilisierte“ Zielgruppe wurden Schüler gewählt, deren Lebenswelt unmittelbar vom Thema School Shootings berührt wird - einerseits durch den Tatort selbst, die Institution Schule, andererseits durch die prinzipiell vorhandene (wenn auch unwahrscheinliche) Gefahr, selbst Opfer eines solchen Ereignisses zu werden und damit verbundene Präventionsmaßnahmen, die als Reaktion auf die Ereignisse in Deutschland mittlerweile von sehr vielen Schulen durchgeführt und von den Schülern miterlebt werden.

4 Vorgespräche (mit Dozenten, Studierenden und Lehrkräften) festigten die Überlegung, in der Gruppendiskussion anstelle des wissenschaftlichen Terminus "School Shooting" den medial geprägten Begriff „Schul-Amoklauf" zu verwenden. Unter diesem Begriff sind die analysierten Ereignisse in der Öffentlichkeit bekannt und es wurde angenommen, dass es den Diskussionsteilnehmern damit leichter fallen würde, über die Thematik zu sprechen. 
tungs- oder Magazinseite nehmen - prozentual gesehen - relativ wenige grafische Elemente ein, nämlich 3,2 Prozent ( $\mathrm{N}=39)$. Insgesamt sind jedoch ein gutes Viertel aller Bilder ( $N=317)$ größer als eine viertel Seite.

\section{Wiederkehrende emotionale und emotionalisierende Bildinhalte}

Eine genauere Betrachtung zeigt, dass sieben zentrale BildInhalte existieren: Täter, Trauernde, Opfer, der Bereich Video/ Film, Experten, Politiker sowie Waffen (vgl. Tab. 1). Unter „Video/Film“ wurden beispielsweise Abbildungen/Screenshots aus Computerspielen (z. B. „Counterstrike“) codiert, aber auch Kinoplakate. Unter „Waffen“ wurden sowohl Fotos als auch Grafiken von Waffen codiert, sowie Bilder, die beispielsweise eine Waffenausstellung (inklusive sichtbarer Waffen) zeigten. „Opfer“ wurde codiert, wenn auf dem Bild entweder ein verletztes oder totes Opfer zu sehen war; dieser Code wurde auch gewählt, wenn es ein Bild war, auf dem das Opfer noch lebend zu sehen war (also z. B. das Bild einer Schülerin aus dem Jahrbuch der Klasse), sofern durch die Bildunterschrift klar war, dass es sich hierbei um eines der Opfer des Shootings handelt.

„Trauernde“ wurde sowohl codiert, wenn eine trauernde Einzelperson zu sehen war (beispielsweise ein weinendes Mädchen, das vor der Schule Blumen ablegt), als auch eine Trauergemeinde (z. B. Foto vom Trauergottesdienst). Ebenfalls wurde dieser Code gewählt, wenn zwar keine Menschen als Trauernde abgebildet waren, diese aber durch den Bildinhalt implizit gemeint waren, beispielsweise das Foto eines Trauerkranzes mit Spruchband, auf dem die Eltern Abschied von ihrer toten Tochter genommen haben. „Experte“ wurde codiert, wenn Wissenschaftler oder Laienexperten abgebildet waren, die aufgrund ihres so bezeichneten oder aus dem Kontext hervorgehenden Expertenwissens zum Sachverhalt befragt worden waren. Der Code „Politiker“ wurde an Personen vergeben, die explizit in dieser Funktion abgebildet waren. Beispielsweise wurde das Foto des damaligen Bundeskanzlers Gerhard Schröder als „Politiker" codiert, auch wenn er in einer Trauersituation zu sehen war.

Wenn der (oder die) Täter auf einem Bild zu sehen waren (auch: Zeichnungen oder grafische Täteranimationen), gab es mehrere Möglichkeiten der Analyse. Zunächst wurde unterschieden, ob es ein „allgemeines“ Abbild des Täter oder ein „selbstinszeniertes“ Bildnis war, also ob der Täter beispielsweise auf einem Schul-, Pass- oder Familienfoto zu sehen war oder ob es ein 


\begin{tabular}{lcc} 
& Häufigkeit & Prozent \\
\hline nicht erkennbar & 1 & 0,1 \\
\hline Trauernde & 134 & 11 \\
\hline Schüler (nicht verletzt) & 58 & 4,8 \\
\hline Opfer (verletzt oder tot) & 122 & 10,1 \\
\hline Schule (real oder Modell) & 37 & 3,1 \\
\hline Umfeld Täter & 22 & 1,8 \\
\hline Befragter Bürger & 31 & 2,6 \\
\hline Experte & 98 & 8,1 \\
\hline Politiker & 92 & 7,6 \\
\hline Video/Film & 107 & 8,8 \\
\hline Symbolbild & 42 & 3,5 \\
\hline Autor & 24 & 2,0 \\
\hline
\end{tabular}

Bild betrifft nicht das hauptsächlich $\begin{array}{lll}\text { thematisierte/gerade erst passierte School } & 22 & 1,8\end{array}$ Shooting, sondern ein anderes

\begin{tabular}{|c|c|c|}
\hline Sonstiges & 149 & 12,3 \\
\hline Evakuierung & 10 & 0,8 \\
\hline Bild Täter allgemein (anonymisiert) & 8 & 0,7 \\
\hline Bild Täter allgemein nicht anonymisiert & 91 & 7,5 \\
\hline Bild Täter selbstinszeniert (anonymisiert) & 4 & 0,3 \\
\hline $\begin{array}{l}\text { Bild Täter selbstinszeniert } \\
\text { (nicht anonymisiert) }\end{array}$ & 44 & 3,6 \\
\hline Bild Täter während der Tat (anonymisiert) & 2 & 0,1 \\
\hline Waffe(n) & 94 & 7,7 \\
\hline $\begin{array}{l}\text { anderes School Shooting: Opfer } \\
\text { (verletzt oder tot) }\end{array}$ & 5 & 0,4 \\
\hline anderes School Shooting: Täter & 16 & 1,3 \\
\hline Gesamt & 1213 & 100,0 \\
\hline Fehlende Werte & 1116 & \\
\hline Gesamt (mit fehlenden Werten) & 2329 & \\
\hline
\end{tabular}

Tab. 1:

Inhalt der den PrintAnalyseeinheiten beigestellten grafischen Elemente/Bilder ( $N=1213$; bis zu fünf erfasste grafische Elemente). 
Foto war, das der Täter entweder selbst von sich gemacht hat oder hat machen lassen - und auf dem er sich selbst in (meist martialischen) Posen und in der Regel mit Waffen darstellt. Eine weitere Darstellungsmöglichkeit war, den Täter „während der Tat“ zu zeigen, beispielsweise durch eine selbstgebaute Grafik oder (wie im Fall von Columbine) durch das Foto eines Fensters, hinter dem die Tätersilhouetten zu sehen sind. In einem zweiten Schritt musste festgestellt werden, ob der Täter anonymisiert gezeigt wird oder nicht, ob er also beispielsweise durch Balken oder Verpixelung unkenntlich gemacht wurde.

Insgesamt zeigen knapp zwei Drittel aller in die Analyse mitaufgenommenen Bilder (65,7 Prozent, $\mathrm{N}=796$ ) entweder den Täter, ein Opfer, ein grafisches Element aus dem Bereich Video/Film, einen Experten, einen Politiker, Trauernde oder Waffen. Die übrigen Bilder oder sonstigen grafischen Elemente $(\mathrm{N}=509)$ verteilten sich auf Schüler $(\mathrm{N}=58)$, Symbolbilder

Die Medien veröffentlichen am

Tatgeschehen beteiligte Personen häufig in emotionalen oder emotionalisierenden Situationen.
$(\mathrm{N}=42)$, die Schule $(\mathrm{N}=37)$, auf befragte Bürger $(\mathrm{N}=31)$, auf den Autor der Analyseeinheit (beispielsweise bei einem Kommentar) $(\mathrm{N}=24)$, auf das Täterumfeld (z. B. Eltern, so bezeichnete Freunde) ( $\mathrm{N}=22)$, auf die Evakuierung (z. B. Kinder, die Rettungsteams entgegenlaufen) ( $\mathrm{N}=10)$ oder auf ein anderes School Shooting allgemein $(\mathrm{N}=22)$ bzw. auf dessen Opfer $(\mathrm{N}=5)$ oder dessen Täter $(\mathrm{N}=16)$.

Im Einzelnen zeigt die Analyse, dass die untersuchten Medien im verwendeten Bildmaterial stark personalisieren und stark emotionalisieren. Sie zeigen am Tatgeschehen direkt und indirekt beteiligte Personen, häufig in emotionalen (z. B. Trauernde) oder emotionalisierenden Situationen (z.B. verletztes Opfer). Gleichzeitig geht von dem Bildmaterial starke Symbolkraft aus - beispielsweise die häufig verwendeten Waffen. Allerdings gibt es hier deutliche Unterschiede zwischen den untersuchten Medien. So wurden 72,9 Prozent der allgemeinen, nicht anonymisierten Täterbilder von der „Bild“-Zeitung veröffentlicht, ebenso wie 47,4 Prozent der selbstinszenierten, nicht anonymisierten Täterbilder. In diesen Bereichen haben sich die Qualitätszeitungen „Süddeutschen Zeitung“ und „Frankfurter Allgemeine Zeitung" zurückgehalten; dies gilt aber nicht für andere Bildinhalte. So wurden beispielsweise 52,7 Prozent $(\mathrm{N}=29)$ der Waffenfotos in der „Süddeutschen Zeitung“ veröffentlicht. An zweiter Stelle ( $\mathrm{N}=11)$ steht hier ebenfalls die „Bild“-Zeitung. 


\section{Selbstinszenierte Täterabbildungen bieten das höchste Identifikationspotenzial}

Während bei den Printbeiträgen die Anzahl und der Inhalt der grafischen Elemente untersucht wurde, wurde bei den TVBeiträgen erfasst, ob während des Beitrags bestimmte Akteure oder Objekte zu sehen waren. Diese waren im Vorfeld aufgrund der qualitativen Analyse und der zu diesem Zeitpunkt bereits laufenden quantitativen Inhaltsanalyse des Printmaterials als Merkmalsausprägungen identifiziert worden - zusätzlich gab es jedoch auch die Möglichkeit, sonstige Akteure bzw. Objekte in einer offenen Kategorie zu erfassen. Dabei zeigte sich, dass „Trauernde bzw. Trauersymbolik“ in mehr als der Hälfte der TV-Beiträge gezeigt wurden (58,3 Prozent, $\mathrm{N}=120)$ und diese Kategorie in der Berichterstattung über School Shootings einen hohen Stellenwert hat. Einen fast ebenso hohen Stellenwert haben "Schüler“, die in 53,4 Prozent der Berichte gezeigt wurden ( $\mathrm{N}=110)$ sowie die "Schule“ (=Tatort) selbst, die in 57,8 Prozent (N=119) der Beiträge vorkommt.

An weiteren Akteuren kommen vor: Experten (51,9 Prozent, $\mathrm{N}=107)$, der Täter selbst (49,5 Prozent, $\mathrm{N}=102)$, Politiker (37,4 Prozent, N=77), Opfer (22,3 Prozent, $\mathrm{N}=46$ ), das Umfeld des Täters (z. B. die Eltern) (14,1 Prozent, N=29), befragte Bürger (7,8 Prozent, $\mathrm{N}=16$ ) sowie Videospiele (als während des Beitrags tatsächlich gezeigtes Spiel, z.B. eine Sequenz aus "Counterstrike“) (12,1 Prozent, N=5). Der Täter, der in knapp der Hälfte aller Beiträge gezeigt wird, wird überwiegend „allgemein“ und „nicht anonymisiert“ abgebildet (76,9 Prozent, $\mathrm{N}=80$ ). Das bedeutet: In den jeweiligen Nachrichtensendungen werden z. B. Familienbilder, Passfotos, Schulfotos oder allgemein im Alltagskontext entstandene Bilder veröffentlicht, die nicht unkenntlich gemacht wurden (die also unverpixelt bzw. ohne schwarzen Balken über den Augen gezeigt werden). Nur drei der „allgemeinen“ Täterdarstellungen waren anonymisiert (2,9 Prozent). Der Anteil der Darstellungen, die vom Täter selbst stammen und als „selbstinszeniert“ bezeichnet wurden, ist mit insgesamt 20,2 Prozent sehr hoch. Davon war ein geringer Teil von fünf Darstellungen (4,8 Prozent) anonymisiert, der größere Teil jedoch (15,4 Prozent, N=16) wurde nicht anonymisiert gezeigt.

Unter selbstinszenierten Darstellungen wurden Bilder und Videos verstanden, die der Täter von sich selbst gemacht hatte und auf denen er sich beispielsweise mit Waffen in Posen zeigte, bei Schießübungen im Wald oder beim Vortragen seiner Ab- 

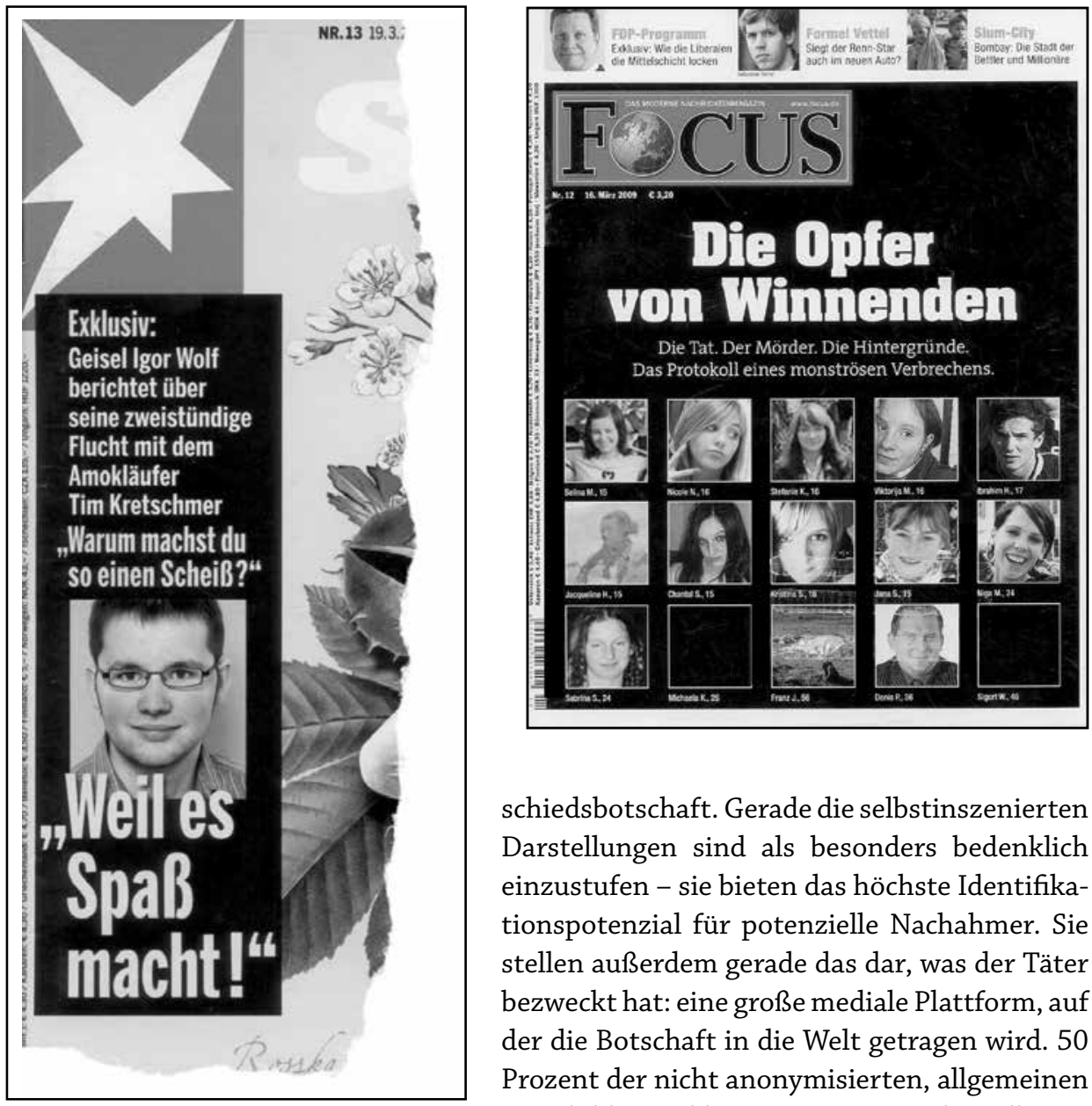

schiedsbotschaft. Gerade die selbstinszenierten Darstellungen sind als besonders bedenklich einzustufen - sie bieten das höchste Identifikationspotenzial für potenzielle Nachahmer. Sie stellen außerdem gerade das dar, was der Täter bezweckt hat: eine große mediale Plattform, auf der die Botschaft in die Welt getragen wird. 50 Prozent der nicht anonymisierten, allgemeinen Täterbilder und knapp 44 Prozent der selbstinszenierten, nicht anonymisierten Täterbilder wurden bei „RTL aktuell“ gezeigt. Selbstinszenierte, anonymisierte Bilder (also beispielsweise das Abschiedsvideo des Täters, bei dem dieser unkenntlich gemacht wurde) wurden ausschließlich bei „ heute“ gezeigt. Die „Tagesschau“ hat im Vergleich zu den anderen Nachrichtensendungen weniger Täterdarstellungen veröffentlicht, hauptsächlich allgemeine, nicht anonymisierte Bilder. Am zweithäufigsten wurden allgemeine, nicht anonymisierte Täterbilder bei „ProSieben“/„Kabel eins“/„Sat.1 Nachrichten“ (27,5 Prozent, $\mathrm{N}=22$ ) und am dritthäufigsten in der Sendung „heute“ gezeigt (16 Prozent, N=13). Die Gruppe „ProSieben“/„Kabel eins“/„Sat.1 Nachrichten" hat außerdem 50 Prozent der selbstinszenierten, nicht anonymisierten Täterbilder gezeigt. Allerdings verteilen sich diese Täterbilder auf die drei zugehörigen Nachrichtensen- 

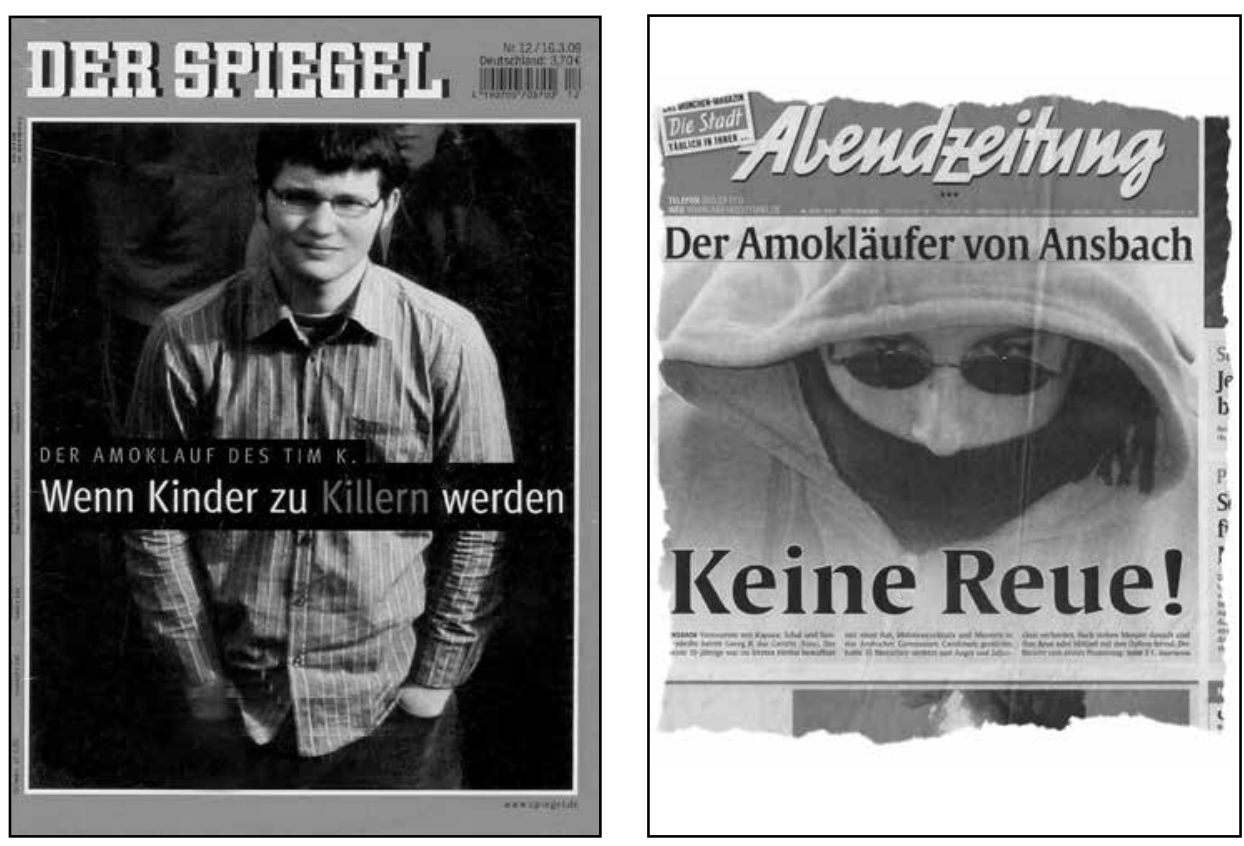

dungen und es ist nicht davon auszugehen, dass ein Rezipient jeweils alle drei Sendungen konsumiert. Insofern sind die sieben (44 Prozent) gezeigten selbstinszenierten Tätervideos und -bilder in der Sendung „RTL aktuell“ aus Wirkungssicht deutlich stärker zu gewichten.

Die offen codierten weiteren Personen oder Objekte, die in den TV-Beiträgen gezeigt wurden, wurden im Nachhinein zu weiteren Kategorien zusammengefasst. Dabei zeigte sich, dass in knapp 15 Prozent aller Beiträge $(\mathrm{N}=29)$ entweder Waffen selbst oder Situationen, in denen Waffen benutzt werden, gezeigt wurden - etwa Beiträge, in denen Schützenvereine thematisiert werden bzw. dort gedreht wurde oder Berichte über eine Waffenausstellung. In jedem zehnten Beitrag $(\mathrm{N}=21)$ kamen sonstige Zeugen oder anderweitig Befragte vor (die keiner anderen oben genannten Gruppe zugeordnet wurden, beispielsweise ein Computerspiel-Fan), in 7,3 Prozent $(\mathrm{N}=15)$ der Beiträge wurde außerdem Archivmaterial zu anderen School Shootings verwendet. In weiteren 14 Fällen (6,8 Prozent) wurden Einsatzfahrzeuge und -helfer gezeigt, Bilder zur Evakuierung der Schule oder beispielsweise Dokumente abgespielt, etwa die Tonbandaufnahme eines Notrufs aus der Bibliothek der Schule. In jedem 20. Beitrag wurden Angehörige der/des Opfer(s) gezeigt, wartende Eltern oder Lehrer (4,9 Prozent).
Abb. 1 bis 4:

Zeitungen und

Magazine präsentieren Täter und Opfer ohne Anonymisierung auf ihren Titelseiten (von links: „Stern“ 13/2009 vom 19.3.; „Focus“ 12/2009 vom 16.3.; „Spiegel“ 12/2009 vom 16.3.; „Abendzeitung München"vom 24.3.2010. 
Rezipienten erinnern die dominierenden Ereignisse und Bilder

Die Befunde zur Bildberichterstattung bei School Shootings spiegeln sich konsistent in den Gruppendiskussionen wider. Insgesamt äußerten sich bei den Gesprächen 89 Schüler_innen und Studierende, die zunächst danach gefragt wurden, was sie mit dem Wort „Schul-Amoklauf“ verbinden, an welche Bilder sie sich möglicherweise erinnern und welche Gedanken ihnen dazu durch den Kopf gehen. Dabei wurden vor allem die Schlagworte genannt, die sich aufgrund der häufig thematisierten Bilder ins Gedächtnis eingebrannt haben. Dazu gehören u. a. „Chaos bzw. chaotische Szenen“ und „Trauernde/Trauersymbolik“, wie Bea (21) plastisch beschreibt:

„Was danach sofort kommt sind diese Massen an Blumen und Bildern und Teddybären, die vor diesen Schulen immer aufgebaut werden, fast ,altarmäßig'sozusagen."

Wie auch Sophia (17), stimmen viele der Schüler_innen zu:

„Also im Fernsehen kommen dann halt meistens Bilder von Trauerfeiern. Wo Leute Kerzen, Blumen und so an Gedenkstätten legen."

Darüber hinaus werden auch Bilder bzw. Filmsequenzen erinnert, die sich einem konkreten School Shooting zuweisen lassen. Vermutlich werden diese Bilder deswegen so häufig erinnert, weil sie auch bei darauffolgenden School Shootings gezeigt und als Archivbilder verwendet wurden. Bert (20):

„Das Erste, was mir da jetzt in den Kopf kommt, ist das Bild, wie Schüler, die in einem Klassenzimmer eingesperrt sind, so ein großes Poster mit "Hilfe" darauf ans Fenster halten."

Auch Akteure, die laut der Diskussionsteilnehmer häufig in der Berichterstattung auftauchen, werden erinnert. Politiker mit „Trauermienen“, wie Lukas (21) beschreibt,

"die sich vor die Kamera stellen und sagen, dass sie dieses Projekt jetzt anpacken werden und dass das jetzt höchste Priorität besitzt".

Oder auch Aktionen von Initiativen, die sich in der Folge der Tat gegründet haben. Ferdinand (19):

„Ich kann mich noch ziemlich gut an so einen Bericht in den Nachrichten erinnern, wo eine Elterninitiative gezeigt wurde, die das richtig inszeniert hat. Die haben einen Müllcontainer aufgestellt und dort Ballerspiele reingeworfen. Und dann sind die richtig darauf rumgetrampelt und so."

Sowohl Schüler_innen als auch Student_innen nennen auf die Frage nach konkreten School Shootings häufig das Schlagwort „Amerika“, beispielsweise Arne (20): 
„Wenn ich an Amokläufe denke, dann denke ich sofort an Amerika, weil leider Gottes ist es nun mal so, dass in Amerika die meisten Amokläufe stattfinden."

Die befragten Schüler_innen erinnern sich aufgrund des geringeren Alters meist nur an die letzte besonders schwerwiegende Tat Winnenden (2009), teilweise auch an Erfurt (2002) und Columbine (1999), an letztere in der Regel aufgrund des Films „Bowling for Columbine“ von Michael Moore, der in manchen Klassen im Unterricht gezeigt wird. Auch die Studierenden erinnern sich an konkrete School Shootings und nennen neben Columbine, Erfurt und Winnenden auch Emsdetten (2006) und Ansbach (2009). In diesem Zusammenhang werden auch Bilder thematisiert, zum Beispiel von Barbara (20):

„Ich muss an Columbine denken, weil wir in der Schule den Film ,Bowling für Columbine' angeschaut haben. Da drin gibt's ja auch diese Videoaufnahmen, ich glaube aus Überwachungskameras oder so, wo man sieht wie in die Mensa reingeschossen wird und die Leute panisch weglaufen oder liegen bleiben im schlimmsten Fall."

\section{Kritik an Journalisten und Berichterstattung}

Die Teilnehmer_innen an den Gruppendiskussionen äußern einhellig sehr klare Kritik an der Herangehensweise vieler Medien bzw. Medienvertreter, die beispielsweise unter Schock stehende Schüler oder Angehörige interviewen oder Eltern, die zum Teil noch nicht wissen, ob ihr Kind überlebt hat, getötet oder verletzt wurde. Arne (20):

„Auch bei der Fernsehberichterstattung werden Leute interviewt und da ist mir immer im Kopf geblieben, dass die einfach mal gar nicht reden können. Die sind einfach so schockiert und das ist vollkommen sinnlos die zu befragen, wie die sich gerade fühlen."

Die Teilnehmer_innen lehnen die ausufernde Berichterstattung, die Bilderflut und die detailgetreue Darstellung ab, wie beispielsweise Moritz (20):

"Ich finde es interessant mal auf Bilder einzugehen, weil ich glaube, dass Bilder sehr viel schneller im Gedächtnis hängen bleiben als ein Text oder ein geschriebenes Wort. Oder eine sehr hohe Zahl an Opfern beispielsweise, die schockierend sein soll, aber wenn man einen blutigen Menschen daliegen sieht, da hat man sofort ein Bild im Kopf."

Die Teilnehmer_innen weisen in diesem Zusammenhang auch darauf hin, dass die Medien durch das Zeigen von Täterbildern möglicherweise genau das tun, was sich dieser erhofft hat. Ferdinand (19): 
„Also ich finde, dass man sich einfach auch fragen muss, ob wirklich alle Bilder gezeigt werden müssen. Zum Beispiel, ob man von dem Täter überhaupt ein Bild zeigen soll. Weil das vielleicht von dem so gewünscht ist, dass er dann dadurch berühmt wird."

Das Zeigen von Bildern wird dabei sowohl in Bezug auf potenzielle Nachahmer diskutiert, als auch in Bezug auf den Täter selbst. Die Schüler_innen und die Studierenden kritisieren hier vor allem, dass der Täter „absichtlich“ negativ dargestellt wird, wie Cemile (17):

„Aber ich denke, es wird von jedem ein Foto geben, wo wir ein bisschen grimmig gucken oder nicht wirklich viel geschlafen haben und so. Und das Foto würden die dann da rausnehmen und zeigen. Und nicht eins, wo man gerade auf einer Party ist und total glücklich aussieht."

Mehrere Äußerungen zeigen außerdem, dass sich die Teilnehmer auch an nachgestellte oder fiktive Darstellungen eindringlich erinnern, wie z. B. Andi (20):

„N24 hat da mal eine Dokumentation über das Columbine Shooting von diesen zwei Freunden gemacht. Und mein Bild von Amokläufern sind die Schauspieler, die das nachgestellt haben. Vor allem wenn es um amerikanische Amokläufe geht, habe ich meistens dieses Bild vor Augen, diese nachgestellten Szenen."

\section{Deutliche Kritik an den Medien}

Die Ergebnisse der Inhaltsanalyse und der Gruppendiskussionen zeigen jeweils, dass eine allgemeine Medienschelte in Bezug auf die (Bild-)Berichterstattung zu School Shootings nicht angebracht ist. Insgesamt werden - in Bezug auf die Print-Berichterstattung - häufiger keine Bilder oder sonstigen grafischen Elemente verwendet. Die meisten Beiträge (63 Prozent) erscheinen also ohne solche Beigaben. Problematisch ist jedoch zu sehen, dass die Medien in dem verwendeten Bildmaterial stark personalisieren und emotionalisieren, wobei nicht anonymisierte Darstellungen des Täters, sowohl alltägliche als auch selbstinszenierte, häufig in der Print- und der TV-Berichterstattung vorkommen. Insgesamt scheinen gerade in der Print-Berichterstattung einige, zahlenmäßig im Vergleich zum Rest nicht übermäßig vertretene Beiträge, die Berichterstattung und das öffentliche Bild davon zu prägen. Das bestätigen auch die Aussagen der Jugendlichen in den durchgeführten Gruppendiskussionen. Problematisch ist außerdem zu sehen, dass insbesondere die langen Beiträge in TV und Print auch prominent platziert werden, also beispielsweise auf der Titelseite einer Zeitung veröffentlicht oder direkt nach dem Schlagzeilenblock einer Nachrichtensendung gebracht wer- 
den. Die damit transportierten Inhalte und Bilder generieren auf diese Weise sehr viel Aufmerksamkeit. Besonders kritisch ist ebenfalls zu sehen, dass der oder die Täter relativ häufig abgebildet werden, abhängig vom jeweiligen Medium auch nicht anonymisiert bzw. in selbstdarstellerischer, selbstinszenierter Pose. In Verbindung damit werden dem Täter viele, teilweise sehr kreative Attribute bzw. Bezeichnungen verpasst, beispielsweise „Babyface-Killer“, „Masken-Mann“ oder „Killermaschine“. Auch die volle Nennung des Vor- und Nachnamens trägt dazu bei, dem oder den Tätern ein identifizierbares Gesicht zu verleihen und darüber Identifikationsmöglichkeiten zu bieten für potenzielle Nachahmer, Bewunderer oder zumindest für Jugendliche, die sich in einer scheinbar ähnlichen Lage befinden.

\section{Empfehlungen für den Umgang mit Bildern}

School Shootings sind zweifelsfrei Ausnahmeereignisse, die alle Beteiligten an die Grenzen ihrer Belastbarkeit bringen. ${ }^{5}$ Klare ethische Verhaltensrichtlinien können hierbei helfen, die Berichterstattung hinsichtlich ethischer Kriterien zu verbessern und gleichzeitig die Verantwortung nicht ausschließlich dem einzelnen Journalisten aufzuladen. Warum es in der referierten Studie zur Berichterstattung über School Shootings auch bei den als „Qualitätsmedien“ ausgewiesenen überregionalen Tageszeitungen und beim öffentlich-rechtlichen Fernsehen zu haarsträubenden Verfehlungen kam, beispielsweise dem Veröffentlichen von Tätervideos, hat mit Sicherheit mehrere Gründe. In jedem Fall geht es immer um Zeit Auch bei den als „Qualitätsmedien“ ausgewiesenen Zeitungen und beim öffentlich-rechtlichen Fernsehen kam es zu haarsträubenden Verfehlungen. und um Geld. Medien unterliegen ökonomischen Zwängen, weil sie sich finanzieren müssen bzw. auch bei gesicherter Finanzierung einem gewissen Quotendruck unterworfen sind - und gleichzeitig in permanenter Konkurrenz zu anderen Medien(unternehmen) stehen.

Der Überlegung, an welchem Punkt ,journalistischer Produktionsprozesse" medienethische Prinzipien Berücksichtigung finden können, liegen zwei unterschiedliche Ansätze zugrunde. 
Aus individualethischer Sicht sollen allgemeingültige Maßstäbe das Handeln von Individuen beeinflussen. Wird jedoch aus systemtheoretischer Sicht argumentiert, müssen Medien als Teil der Gesellschaft in den Blick genommen werden. Geht man noch einen Schritt weiter, müssen auch Standesethik und Publikumsethik Berücksichtigung in der medienethischen Debatte finden (vgl. Schicha 2010, S. 34). Grund-

Der Deutsche Presserat hat einen Leitfaden entworfen, an dem sich Journalisten bei der Berichterstattung über School Shootings orientieren können. sätzlich geht es also darum, wer Verfehlungen im Journalismus verantworten muss: Der einzelne Journalist, dessen Rollenverantwortung in der Berichterstattung zum Ausdruck kommen muss (vgl. ebd., S. 35)? Das

Medienunternehmen? Gesellschaft und Politik an sich, weil die Rahmenbedingungen für Journalisten nicht stimmen oder weil es keine oder mangelhafte Maßstäbe gibt, an denen sich Journalisten orientieren könnten? Oder doch der einzelne Leser, Hörer oder Zuschauer, der schließlich selbst darüber entscheidet, welche Medieninhalte er oder sie konsumiert und damit auch dafür verantwortlich ist, was produziert wird?

In Bezug auf School Shootings kann es, auch wenn dies aus rein wissenschaftlicher Sicht und unter Berücksichtigung der empirischen Studien hierzu zu begrüßen wäre, nicht darum gehen, überhaupt nicht $\mathrm{zu}$ berichten. Schließlich handelt es sich um Ereignisse von großer Tragweite und Relevanz, und eine wichtige Aufgabe von Medien ist es, die Öffentlichkeit umfassend $\mathrm{zu}$ informieren und dem legitimen Informations- und Leserinteresse nachzukommen. Daher kann es bei einer medienethischen Entscheidung bezüglich einer Berichterstattung über School Shootings nicht darum gehen, ob berichtet wird, sondern nur wie berichtet wird.

In der Folge seiner Entscheidungen zu den zahlreichen Beschwerden über die Berichterstattung zur Tat von Winnenden hat der „Deutsche Presserat“ einen Leitfaden für Redaktionen entworfen, an dem sich die Journalisten bei ihrer Berichterstattung über School Shootings und Amokläufe orientieren können. ${ }^{6}$ Dies ist als wichtiger Schritt zu begrüßen, weil es den einzelnen Journalisten zwar nicht von seiner Verantwortung entbindet, gleichzeitig aber die Medienunternehmen im Allgemeinen in den Blick rückt. Daher richtet sich der Leitfaden auch ausdrück-

6 Der Leitfaden wird derzeit überarbeitet und steht daher nicht zum Download zur Verfügung. 
lich sowohl an einzelne Journalisten, als auch an Redaktionen. Diese sehr positive Entwicklung ist allerdings auch gewissen Einschränkungen unterworfen. Erstens gibt es für den „Deutschen Presserat" keine rechtliche Handhabe, Verstöße gegen diese Empfehlungen zu ahnden. Zweitens sind die Empfehlungen selbst noch zu sehr an der Grenze des Erlaubten und zu wenig an der Grenze des Wünschenswerten. Beispielsweise sollte es eine Mindestforderung sein, den Täter immer anonymisiert abzubilden und niemals in selbstinszenierten Posen - denn die mögliche Schlussfolgerung, dass der Täter durch sein Handeln zu einer relativen Person der Zeitgeschichte geworden ist (vgl. Deutscher Presserat 2010, S. 3), rechtfertigt diese Abbildung angesichts der Bedeutung für potenzielle Nachahmungstäter nicht. Idealerweise wird dem bzw. den Täter(n) also weder ein Gesicht gegeben noch ein (voller) Name bzw. Synonyme (z. B. „Killer"), der im Gedächtnis haften bleibt. Die Minimalforderung des „Deutschen Presserates" sollte lauten, ohne Ausnahme keinerlei Bildmaterial zu veröffentlichen, das von den Tätern selbst stammt, also inszeniert ist (beispielsweise Posieren mit Waffen).

Auch im Hinblick auf die Opfer solcher Taten gibt es Verbesserungsmöglichkeiten am Leitfaden des "Deutschen Presserates“. Die bisherige Fassung weist darauf hin, dass die Veröffentlichung von Porträtfotos der Opfer im Einzelfall und im Kontext einer sachlichen Dokumentation erlaubt sein könne, der vollständige Name jedoch nicht genannt werden dürfe (Ziffer 3, S. 3). Auch die Nennung persönlicher Details (Beziehung, Hobbies etc.) sei nicht zulässig, ebenso das Herausheben eines einzelnen Opfers ohne Zustimmung der Angehörigen (Ziffer 3, S. 3). Dennoch verweist der „Deutsche PresseIn Bezug auf die Opfer und deren Familien sollten keinerlei Porträtfotos oder Fotos erkennbarer Einzelpersonen verwendet werden. rat" wie schon beim Täter auf die Ausnahme in Bezug auf Personen der Zeitgeschichte. Diese Ausnahme ist aus Informationsinteresse verständlich, ermöglicht den Medien jedoch ein Schlupfloch, mit dem viel begründet werden kann. In Bezug auf Opfer und Opferfamilien sollten aus diesem Grund keinerlei Porträtfotos bzw. Fotos erkennbarer Einzelpersonen verwendet werden. Dadurch können Opfer allgemein geschützt, Re-Traumatisierung vermieden und dem Entstehen von Heldenmythen vorgebeugt werden. Abgesehen davon ist die Namensnennung und Abbildung von Familienangehörigen und sonstigen durch die Veröffentlichung mittelbar Betroffene, die mit dem Unglücksfall oder der Straftat nichts zu tun haben, grundsätzlich unzulässig (Richtlinie 8.1 (3), S. 4). Wird jedoch der volle Name 
Abb. 5: Richtigstellung in der „Bild“-Zeitung vom 14.3.2009.

Nach dem School Shooting von Winnenden publizierten Medien ein Foto des Täters - tatsächlich zeigte das Bild einen Namensvetter.

\section{Fotoverwechslung!}

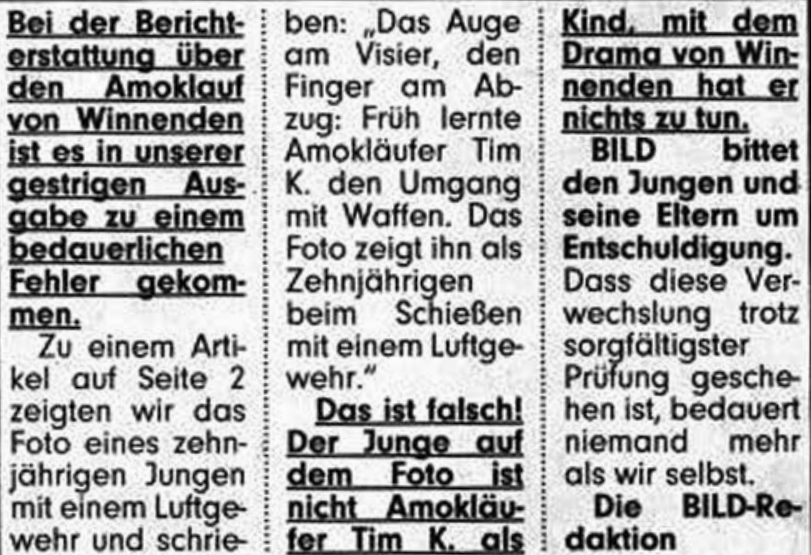

des Täters genannt, karikiert sich diese Richtlinie des Leitfadens selbst, denn oftmals haben die Täter Geschwister, die durch diese Namensnennung ebenfalls bekannt werden und für die das weitreichende Konsequenzen hat. Schon aus diesem Grund sollte auf die volle Namensnennung des Täters verzichtet werden. Ein zweiter Grund wird durch ein Beispiel anschaulich illustriert. Am Tag des School Shootings von Winnenden war der Name des Täters, Tim K., relativ schnell bekannt - ebenso wie der 21-jährige Namensvetter Tim K., ein IT-Fachmann aus Bremen, der von den Medien ausfindig gemacht und zum „Amokschützen“ ausgerufen

wurde (vgl. Abb. 5). Sein Foto von der Firmenhomepage ging über Twitter um die Welt (vgl. Verhovnik 2009, S. 291). Wie ein plakatives Beispiel zeigt, ist eine alternative Berichterstattung in Bezug auf Opfer (und auch Täter) nicht nur möglich, sondern kann sich sogar lohnen. Die „Winnender Zeitung“ machte sich beim School Shooting von Winnenden (2009) eine zurückhaltende Haltung zu Eigen - ohne Opferfotos, ohne Beerdigungsberichte und mit einem behutsamen Umgang mit Stellungnahmen traumatisierter Kinder (vgl. ebd., S. 292). Aufgrund dieser Haltung kamen die Familien der Opfer nach kurzer Zeit selbst auf die Zeitung zu: 
„After a while a number of families contacted the paper, because they wanted the newspaper to convey their experience to other members of the community, and in particular to tell their neighbours and acquaintances that they wanted to be approached and talked to" (Rees 2012, S. 340).

\section{Literatur}

Addington, Lynn A. (2009): Cops and Cameras. Public School Security as a policy response to Columbine. American Behavioral Scientist, 52. Jg., Nr. 10, S. 1426-1446.

Associated Press (2009): Columbine High School Marks 10 Years Since Massacre on Monday. In: Associated Press vom 17.9. http://www.foxnews.com/ story/2009/04/17/columbine-high-school-marks-10-years-since-massacreon-monday/ (zuletzt aufgerufen am 20. 12. 2013).

Bannenberg, Britta (2010): Amok. Ursachen erkennen - Warnsignale verstehen - Katastrophen verhindern. Gütersloh.

Böckler, Nils/Seeger, Thorsten/Sitzer, Peter (2012): Media dynamics in school shootings: a socialization theory perspective. In: Muschert, Glenn W./Sumiala, Johanna (Hg.): School Shootings: Mediatized Violence in a Global Age. Bingley, S. 25-46.

Böckler, Nils/Seeger, Thorsten (2010): Schulamokläufer. Eine Analyse medialer Täter-Eigendarstellungen und deren Aneignung durch jugendliche Rezipienten. Weinheim, München.

Bondü, Rebecca (2012): School Shootings in Deutschland: Internationaler Vergleich, Warnsignale, Risikofaktoren, Entwicklungsverläufe. Diss. Univ. Berlin.

Deutscher Presserat (2010): Praxis-Leitfaden. Berichterstattung über Amokläufe - Empfehlungen für Redaktionen. Berlin.

Engels, Holger (2007): Das School Shooting von Emsdetten - der letzte Ausweg aus dem Tunnel?! Eine Betrachtung aus Sicht des Leiters der kriminalpolizeilichen Ermittlungen. In: Hoffmann, Jens (Hg.): Amok und zielgerichtete Gewalt an Schulen. Früherkennung, Risikomanagement, Kriseneinsatz, Nachbetreuung. Frankfurt am Main, S. 35-56.

Larkin, Ralph J. (2007): Comprehending Columbine. Philadelphia.

Lindgren, Simon (2012): Collective coping through networked narratives: Youtube responses to the Virginia Tech Shooting. In: Muschert, Glenn W.I Sumiala, Johanna (Hg.): School Shootings: Mediatized Violence in a Global Age. Bingley, S. 279-298.

Meier, Urs (2007): Das Mediensystem im Zeitalter seiner wirtschaftlichen Kalkulierbarkeit. Terroristen und Amokläufer steuern zunehmend die Berichterstattung über ihre Taten. In: Medienheft vom 23.4, S. 1-2.

Moore, Mark H. et al. (Hg.) (2003): Deadly Lessons. Understanding Lethal School Violence. Washington, D.C. 
Muschert, Glenn W. (2013): School Shootings as Mediatized Violence. In: Böckler, Nils et al. (Hg.): School Shootings. International research, case studies, and concepts for prevention. New York, S. 265-281.

Muschert, Glenn/Larkin, Ralph J. (2007): The Columbine High School Shootings. In: Bailey, Frankie/ Chermak, Steven (Hg.): Crimes and Trials of the Century. Westport, CT, S. 253-266.

Newman, Katherine S. (2004): Rampage. The social roots of school shootings. New York.

Pew Research Center for the People and the Press. (1999). Columbine shooting biggest news draw of 1999. http://www.people-press.org/1999/12/28/ columbine-shootingbiggest-news-draw-of-1999/ (zuletzt aufgerufen am 26.11.2014).

Polizeidirektion Waiblingen, B.-W. (2009). Winnenden/Wendlingen/Waiblingen, Mai 2009. Vorläufiges Ergebnis zum Amoklauf liegt vor. Gemeinsame Pressemitteilung der Staatsanwaltschaft Stuttgart und der Polizeidirektion Waiblingen. http://org.polizei-bwl.de/PDWaiblingen/Presse/PM/PMProzent20AmokProzent20EndfassungProzent20standProzent20MitteProzent20Mai.pdf (zuletzt aufgerufen am 22. 8. 2013).

Rees, Gavin (2012): Afterword: Is mediatization a useful concept for informing practice in journalism? In: Muschert, Glenn W./Sumiala, Johanna (Hg.): School Shootings: Mediatized Violence in a Global Age. Bingley, S. 333-341.

Robertz, Frank (2004). School shootings. Über die Relevanz der Phantasie für die Begehung von Mehrfachtötungen durch Jugendliche. Frankfurt am Main.

Schicha, Christian/ Brosda, Carsten (Hg.) (2010): Handbuch Medienethik. Münster.

Sitzer, Peter (2013): The Role of Media Content in the Genesis of School Shootings: The Contemporary Discussion. In: Böckler, Nils et al. (Hg.): School Shootings. International research, case studies, and concepts for prevention. New York, S. 283-307.

Szumelda, Christoph/Behling, Yvonne (2007): Die Columbine Erben - Wie Bastian B. den Freitod wählte. Hamburg.

Twemlow, Stuart W. et al. (2002): Premeditated Mass Shootings in Schools: Threat Assessment. American Academy of Child \& Adolescent Psychiatry, 41 Jg., Nr. 4, S. 475-477.

Verhovnik, Melanie (2015, im Druck): School Shootings. Interdisziplinäre Analyse und Darstellung der journalistischen Berichterstattung. Baden-Baden.

Verhovnik, Melanie (2009): Chaos vom Feinsten. Berichterstattung zum Amoklauf in Winnenden. Communicatio Socialis, 42. Jg., Nr. 3, S. 288-292.

Weirauch, Wolfgang/Buermann, Uwe (2010): Kollektives Ekeltraining. Interview mit Uwe Buermann. In: Flensburger Hefte (Hg.): Leere Seelen. Was treibt sie in den Amok? Flensburg, S. 63-110. 